\title{
Physical Aging and Light Scattering of Low-Loss Poly(methyl methacrylate) Glass
}

\author{
Norihisa TANIO, ${ }^{*}$ Hisao Kato, ${ }^{* *}$ Yasuhiro KoIKE, ${ }^{* * *}$ Harvey E. BaIR, ${ }^{* * *}$ \\ Shiro MatsuOKa, ${ }^{* * *, \dagger}$ and Lee L. BLyLER, Jr.*** \\ * Kanagawa Academy of Science and Technology, \\ 1-1-1 Fukuura, Kanazawa-ku, Yokohama 236, Japan \\ ** Faculty of Science and Technology, Keio University, \\ 3-14-1 Hiyoshi, Kohoku-ku, Yokohama 223, Japan \\ *** Bell Laboratories, 700 Mountain Avenue, Murray Hill, NJ 07974, U.S.A.
}

(Received June 26, 1997)

\begin{abstract}
Isothermal aging has been carried out on low-loss poly(methyl methacrylate) (PMMA) glass at $18^{\circ} \mathrm{C}$ below the glass transition temperature $\left(T_{\mathrm{g}}\right)$. The effect of enthalpy relaxation on light scattering loss of PMMA glass which had no excess scattering was investigated by the use of differential scanning calorimeter (DSC) and light scattering measurement. The light scattering intensity of PMMA glass did not increase with increasing aging time. It is seen that the light scattering loss of glassy PMMA is not affected inherently by physical aging as a result of its approach to thermodynamic equilibrium at a temperature $18^{\circ} \mathrm{C}$ below its $T_{\mathrm{g}}$.

KEY WORDS Physical Aging / Light Scattering Loss / Poly(methyl methacrylate) / Glass Transition Temperature / Enthalpy Relaxation / Amorphous Polymer / Polymer Glass /
\end{abstract}

Polymer glasses are essentially thermodynamic nonequilibrium states. Physical aging ${ }^{1}$ is the term used to describe the time dependence of changes in the behavior of a polymer glass held at temperatures below the glass transition; such changes are normally the result of continuous slow relaxation of the glass from its initial non-equilibrium state towards its final thermodynamic equilibrium state. The thermodynamical properties such as volume $e^{2,3}$ or enthalpy ${ }^{4,5}$ have been mainly considered for the study of this phenomenon. After a quench into the glassy state, the polymer shows a continuous decrease of the specific volume with time, which leads to a decrease in free volume and thus of mobility of the polymer molecules. This change of mobility of the molecular segments is assumed to be the origin of the change in dynamic mechanical properties during aging. 6 Many other properties of amorphous polymer glasses can be affected by aging. However, the effect of isothermal aging, and the resulting densification, on the optical properties of amorphous polymer glasses has not been studied well.

Poly(methyl methacrylate) (PMMA) glass is a common optical amorphous polymer and has been already used as a core material of polymer optical fiber (POF). The two important intrinsic loss factors in POF are $\mathrm{C}-\mathrm{H}$ overtone absorption and Rayleigh scattering due to density fluctuations. Recently, we demonstrated that the light scattering loss of PMMA glasses, even in using contaminant-free monomer varied widely with polymerization and heat-treatment conditions. ${ }^{7-11}$ PMMA glasses polymerized below the glass transition temperature $\left(T_{\mathrm{g}}\right)$ had excess scattering loss (hundreds $\mathrm{dB} \mathrm{km}^{-1}$ ) due to large-size (several hundreds $\AA$ ) heterogeneous structure with the order of $10^{-5}-10^{-4}$ of refractiveindex difference. On the other hand, with sufficient heat-treatment above the $T_{\mathrm{g}}$, this size heterogeneity almost completely disappeared and the isotropic light scattering loss was reduced to $9.7 \mathrm{~dB} \mathrm{~km}^{-1}(633 \mathrm{~nm})$ which is close to the value $\left(9.5 \mathrm{~dB} \mathrm{~km}^{-1}\right)$ predicted from the thermally induced fluctuation theory. ${ }^{12}$ The glass transition temperature is the critical temperature for determining the excess light scattering of PMMA glasses.

In this study, isothermal aging has been carried out on low-loss PMMA glass at $18^{\circ} \mathrm{C}$ below $T_{\mathrm{g}}$. The effect of enthalpy relaxation on light scattering loss of PMMA glass which had no excess scattering was investigated as it approached equilibrium by the use of differential scanning calorimeter (DSC) and light scattering measurement.

\section{EXPERIMENTAL}

\section{Preparation of PMMA Bulk}

After an ordinary purification procedure of methyl methacrylate (MMA) monomer with a distillation (bp $46-47^{\circ} \mathrm{C} / 100 \mathrm{mmHg}$ ) and $0.2 \mu \mathrm{m}$ membrane filter, a rigorous purification was carried out as follows: Ampoule A with the distilled monomer was connected with two ampoules B and C carefully purified. Here, di-tert-butyl peroxide (DBPO) as an initiator and $n$-butyl mercaptan $(n \mathrm{BM})$ as a chain transfer agent were placed in ampoule $\mathrm{B}$ and ampoule $\mathrm{C}$ was empty. Ampoules $\mathrm{A}$ and $\mathrm{B}$ were frozen with liquid nitrogen, evacuated, and substituted by nitrogen. Then the monomers, DBPO, and $n \mathrm{BM}$ were degassed by several freeze-thaw cycles, and slowly distilled into the ampoule $\mathrm{C}$ under vacuum by cooling ampoule $\mathrm{C}$ with liquid nitrogen. Ampoule $\mathrm{C}$ was sealed under vacuum and immersed in silicone oil for polymerization. After polymerization, the resulting cylindrical polymer sample with a $20 \mathrm{~mm}$ diameter was removed from ampoule $\mathrm{C}$ for measurement.

\footnotetext{
${ }^{\dagger}$ Present address: Polytechnic University, Brooklyn, NY 11201, U.S.A.
} 


\section{Light Scattering Measurement}

Light scattering measurements were made with light scattering spectrophotometer (Otsuka Electronics, DLS7000). The details of theory and analytical procedure of light scattering were described elsewhere. ${ }^{7}$ The sample was placed in the center of the cylindrical glass cell. The gap between the sample and inner wall of the glass cell was filled with immersion oil with a refractive index of 1.5. This glass cell was perpendicularly located at the center of the goniometer, and a parallel beam of vertically polarized $\mathrm{He}-\mathrm{Ne}$ laser (wavelength $\lambda_{0}=633 \mathrm{~nm}$ ) was injected from the side. In this paper, polarized $\left(V_{\mathrm{V}}\right)$ and depolarized $\left(H_{\mathrm{V}}\right)$ scattered intensities were measured at room temperature. Here the letters $H$ and $V$ ( or $_{\mathrm{v}}$ ) denote horizontal and vertical polarization, respectively, and the upper case letter and the subscript denote the scattered and the incident beam, respectively. To estimate the absolute intensity, pure benzene purified in the same manner as the monomer mixture mentioned above was used as a standard for calibration. The measurement error of the scattering intensity $\left(V_{\mathrm{V}}\right.$ or $\left.H_{\mathrm{V}}\right)$ was less than $2 \%$.

In order to analyze the local structure, we separated $V_{\mathrm{V}}$ into the two terms of eq 1 .

$$
V_{\mathrm{v}}=V_{\mathrm{v} 1}+V_{\mathrm{v} 2}
$$

where $V_{\mathrm{V}_{1}}$ denotes the background intensity independent of the scattering angle, and $V_{\mathrm{V} 2}$ denotes the excess scattering with angular dependence due to large size heterogeneities. In a structureless liquid or randomly oriented polymer bulk, the isotropic part $V_{\mathrm{V} 1}$ iso of $V_{\mathrm{V} 1}$ is given by eq 2 .

$$
V_{\mathrm{V} 1}{ }^{\text {iso }}=V_{\mathrm{V} 1}-(4 / 3) H_{\mathrm{V}}
$$

Here, the $H_{\mathrm{v}}$ intensity for our sample examined in this paper had no angular dependence (see ref 7).

Scattering loss was estimated by integrating the scattering intensities in all directions. The total light scattering loss $\alpha_{\mathrm{t}}\left(\mathrm{dB} \mathrm{km}^{-1}\right)$ is composed of three terms, i.e., $\alpha_{1}{ }^{\text {iso }}, \alpha_{2}{ }^{\text {iso }}$, and $\alpha^{\text {aniso }}$. Here $\alpha_{1}{ }^{\text {iso }}$ is the loss due to the isotropic $V_{\mathrm{V} 1}$ iso scattering without angular dependence, $\alpha_{2}{ }^{\text {iso }}$ is due to the isotropic $V_{\mathrm{V} 2}$ scattering with angular dependence, and $\alpha^{\text {aniso }}$ is due to anisotropic scattering, $H_{\mathrm{v}}$.

$$
\begin{gathered}
\alpha_{1}{ }^{\text {iso }}\left(\mathrm{dB} \mathrm{km}^{-1}\right)=1.16 \times 10^{6} \pi V_{\mathrm{v} 1} \text { iso } \\
\alpha_{2}{ }^{\text {iso }}\left(\mathrm{dB} \mathrm{km}^{-1}\right)=\frac{1.35 \times 10^{9} a^{3}\left\langle\eta^{2}\right\rangle}{n^{4} \lambda^{4}} \\
\times\left[\frac{(b+2)^{2}}{b^{2}(b+1)}-\frac{2(b+2)}{b^{3}} \ln (b+1)\right] \\
b=\frac{16 \pi^{2} a^{2}}{\lambda^{2}} \\
\alpha^{\text {aniso }}\left(\mathrm{dB} \mathrm{km}^{-1}\right)=3.86 \times 10^{6} \pi H_{\mathrm{v}}
\end{gathered}
$$

Equation 4 is obtained using Debye's theory. ${ }^{13,14}$ Here symbol $a(\AA)$ is called the correlation length and is a measure of the size of the heterogeneous structure inside the bulk. Symbol $\left\langle\eta^{2}\right\rangle$ denotes the mean-square average of the fluctuation of all dielectric constants, $n$ is the refractive index of medium, and $\lambda$ is the wavelength of light in a medium.

\section{Enthalpy Relaxation Measurement}

The enthalpy relaxation of polymer glasses was measured by means of DSC (Shimadzu Co., DSC-50). The following standard thermal history was employed for the low-loss PMMA sample:

1) In order to eliminate the effects of previous thermal history after polymerization, the PMMA sample was annealed at $110^{\circ} \mathrm{C}$ for $3 \mathrm{~h}$.

2) The PMMA sample was annealed for an aging time of $t_{\mathrm{a}}$ at the chosen aging temperature $T_{\mathrm{a}}$ (in this study, $\left.T_{\mathrm{a}}=90^{\circ} \mathrm{C}\right)$.

3) The PMMA sample was quenched down to the room temperature.

4) Then DSC measurements were carried out on increasing temperature from room temperature to $170^{\circ} \mathrm{C}$ at a heating rate of $10^{\circ} \mathrm{C} \mathrm{min}^{-1}$.

\section{Other Measurement}

The molecular weight was measured by a gel permeation chromatography (GPC) (Tosoh Co., HLC8120 GPC) with tetrahydrofuran (THF) as a carrier. The densities were compared by preparing aqueous solutions of calcium nitrate and determining whether test specimens sank or floated in these standard solutions. ${ }^{15}$

\section{RESULTS AND DISCUSSION}

The total light scattering loss of PMMA glasses varies widely with polymerization conditions from $c a$. 13 $\mathrm{dB} \mathrm{km}{ }^{-1}$ to $c a .800 \mathrm{~dB} \mathrm{~km}^{-1}$ at $\lambda_{0}=633 \mathrm{~nm} .^{7-11}$ With polymerization above the $T_{\mathrm{g}}$, the isotropic light scattering loss was reduced to $9.7 \mathrm{~dB} \mathrm{~km}^{-1}(633 \mathrm{~nm})$ which is close to the value $\left(9.5 \mathrm{~dB} \mathrm{~km}^{-1}\right)$ predicted from the thermally induced fluctuation theory. ${ }^{12}$ The intensity of the isotropic light scattering $\left(V_{\mathrm{V} 1}\right.$ iso $)$ from thermally induced density fluctuations in a structureless liquid is written as, ${ }^{12}$

$$
V_{\mathrm{V} 1} \text { iso }=\frac{\pi^{2}}{9 \lambda_{0}{ }^{4}}\left(n^{2}-1\right)^{2}\left(n^{2}+2\right)^{2} k T \beta
$$

where $\lambda_{0}$ is the wavelength of light in vacuum, $k$ is the Boltzmann constant, $T$ is the absolute temperature, $n$ is the refractive index, and $\beta$ is the isothermal compressibility. The fluctuation theory for structureless liquids indicates that according to eq 6 , the isotropic scattering loss decreases with the decrease in isothermal compressibility and refractive index. The value of $V_{\mathrm{V} 1}{ }^{\text {iso }}$ calculated using the value of $\beta$ at $T_{\mathrm{g}}$ according to a frozen model was good agreement with the observed value for PMMA glass. ${ }^{8}$ In this study, the low-loss PMMA glass with no excess scattering (its isotropic light scattering loss at $633 \mathrm{~nm}$ is $9.7 \mathrm{~dB} \mathrm{~km}^{-1}$ ) was used for calorimetric and light scattering measurement. This PMMA sample $\left(M_{w}=7.0 \times 10^{4}, M_{w} / M_{n}=2.1\right)$ was polymerized at $130^{\circ} \mathrm{C}$ for $96 \mathrm{~h}$ and the weight percentages of DBPO and $n \mathrm{BM}$ were each $0.20 \mathrm{wt} \%$.

The heat capacity of a polymer glass undergoes a step change in the glass transition region. We have defined the glass transition temperature, $T_{\mathrm{g}}$, as the temperature at which the change of heat capacity associated with glass transition is $\Delta C_{p} / 2$, where $\Delta C_{p}$ is the specific heat 


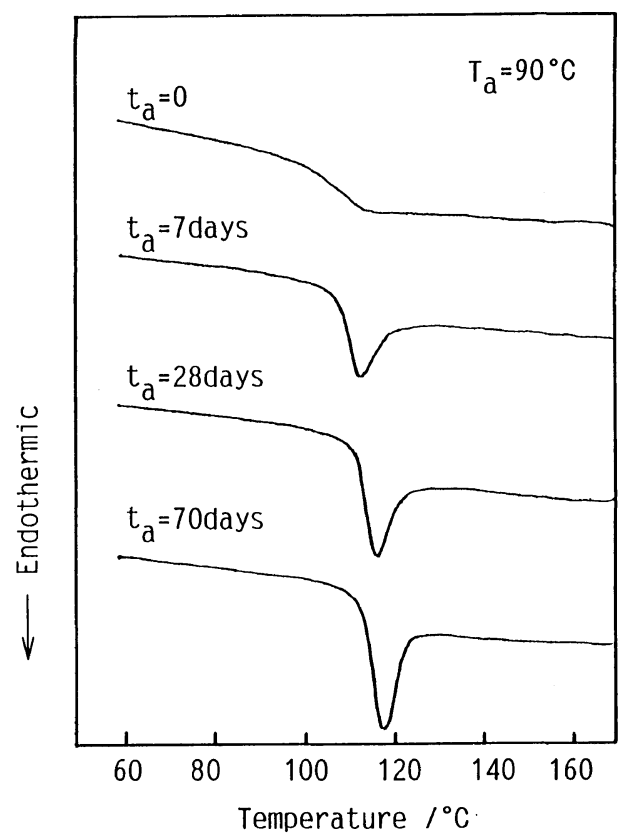

Figure 1. DSC curves of PMMA glasses aged at $90^{\circ} \mathrm{C}$.

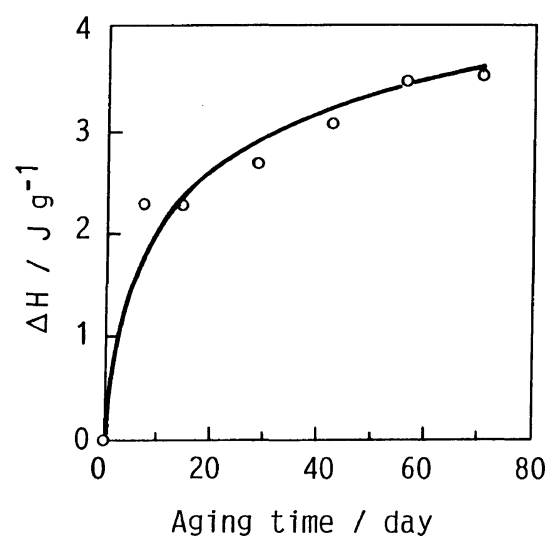

Figure 2. Enthalpy relaxation $(\Delta H)$ plotted as a function of aging time for PMMA glass at $90^{\circ} \mathrm{C}$.

increment between the liquid and glass states at glass transition region. The value of $T_{\mathrm{g}}$ for unaged low-loss PMMA glass was $108^{\circ} \mathrm{C}$.

Physical aging is the phenomenon which is known to be time- and temperature-dependent. ${ }^{16}$ Most of the studies on physical aging for amorphous polymers have been performed at temperatures close to $T_{\mathrm{g}}$. We measured the enthalpy relaxation for low-loss PMMA glass at temperatures, $T_{\mathrm{g}}-28^{\circ} \mathrm{C}, T_{\mathrm{g}}-18^{\circ} \mathrm{C}$, and $T_{\mathrm{g}}-8^{\circ} \mathrm{C}$. The amounts of enthalpy relaxation was $3.3,3.8$, and 1.8 $\mathrm{J} \mathrm{g}^{-1}$, respectively. The maximum value of enthalpy relaxation was found at $T_{\mathrm{g}}-18^{\circ} \mathrm{C}$. Therefore, in this work, we have studied isothermal aging effects occurring at $18^{\circ} \mathrm{C}$ below $T_{\mathrm{g}}$. DSC studies of the enthalpy relaxation process for low-loss PMMA glasses of different aging times at $90^{\circ} \mathrm{C}\left(=T_{\mathrm{g}}-18^{\circ} \mathrm{C}\right)$ are illustrated in Figure 1. Aging at $90^{\circ} \mathrm{C}$ produces an endothermic peak at the glass transition temperature. When aging times increase, the endothermic peak increases. From these thermograms, enthalpy relaxation $\Delta H$ can be determined by taking the difference between the curve corresponding to the quenched state and the curves corresponding to different aged states. Figure 2 presents the evolution of $\Delta H$ as a

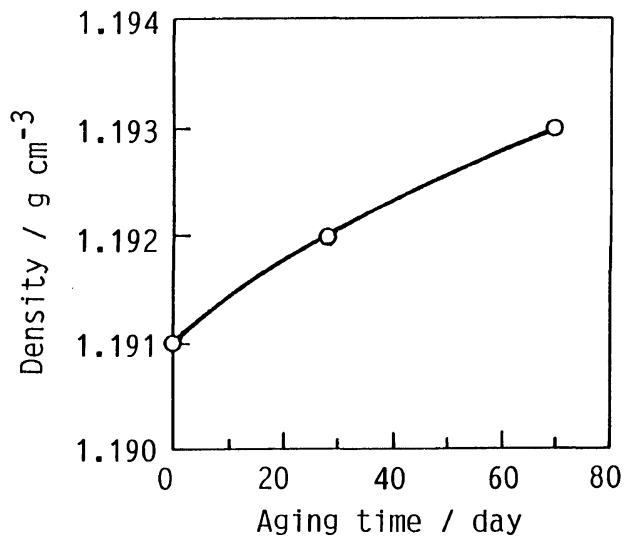

Figure 3. Density plotted as a function of aging time for PMMA glass at $90^{\circ} \mathrm{C}$.

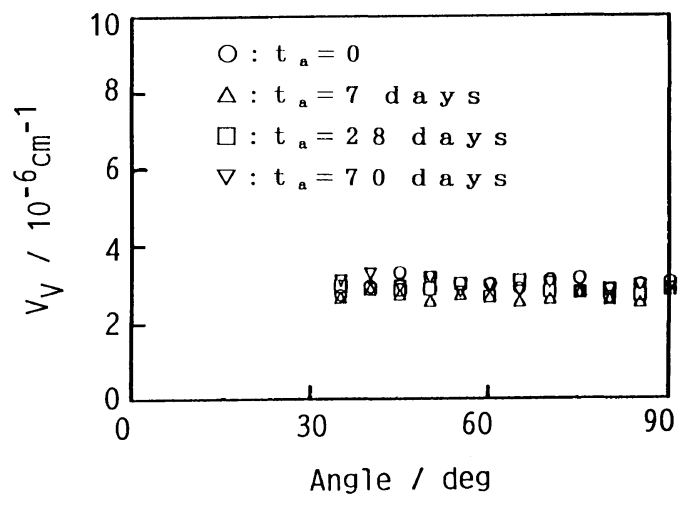

Figure 4. $V_{\mathrm{v}}$ scattering by PMMA glasses aged at $90^{\circ} \mathrm{C}$.

Table I. Light scattering losses of PMMA glasses aged at $90^{\circ} \mathrm{C}$

\begin{tabular}{|c|c|c|c|c|c|}
\hline $\begin{array}{l}\text { Aging } \\
\text { time, } t_{\mathrm{a}}\end{array}$ & $\Delta H$ & $\alpha_{1}{ }^{\text {iso }}$ & $\alpha_{2}{ }^{\text {iso }}$ & $\alpha^{\text {aniso }}$ & $\alpha_{t}$ \\
\hline days & $\mathrm{Jg}^{-1}$ & $\mathrm{~dB} \mathrm{~km}^{-1}$ & $\mathrm{~dB} \mathrm{~km}{ }^{-1}$ & $\mathrm{~dB} \mathrm{~km}^{-1}$ & $\mathrm{~dB} \mathrm{~km}^{-1}$ \\
\hline 0 & 0 & 9.7 & 0 & 2.8 & 12.6 \\
\hline 7 & -2.5 & 8.7 & 0 & 2.6 & 11.3 \\
\hline 28 & -3.1 & 9.3 & 0 & 2.6 & 12.0 \\
\hline 70 & -3.8 & 9.6 & 0 & 2.7 & 12.3 \\
\hline
\end{tabular}

function of aging time for an aging temperature of $90^{\circ} \mathrm{C}$.

The density measurements for low-loss PMMA glass were performed with isothermal aging at $90^{\circ} \mathrm{C}$. The result is shown in Figure 3. Before aging, the value of density was 1.191. With increasing time, the density increased, and reached to 1.193 at 70 days. This increase is caused by the densification due to the aging effect below $T_{\mathrm{g}}$, which was recognized by a large enthalpy relaxation peak just after the $T_{\mathrm{g}}$ in Figure 1 when increasing the temperature.

In order to rigorously confirm the effect of the densification on the excess scattering, polarized $\left(V_{\mathrm{V}}\right)$ and depolarized $\left(H_{\mathrm{V}}\right)$ scattering intensities were measured for aged PMMA samples. Figure 4 shows $V_{\mathrm{V}}$ scattering for unaged and aged PMMA glasses. Before aging, the isotropic $V_{\mathrm{V}}$ scattering had no angular dependence and isotropic light scattering loss, $\alpha_{1}{ }^{\text {iso }}$ value was $9.7 \mathrm{~dB} \mathrm{~km}^{-1}$ as shown in Table I. With increasing aging time, the isotropic light scattering $V_{\mathrm{V}}$ did not increase, and $\alpha_{1}$ iso value at 70 days was $9.6 \mathrm{~dB} \mathrm{~km}^{-1}$. Figure 5 shows $H_{\mathrm{v}}$ 


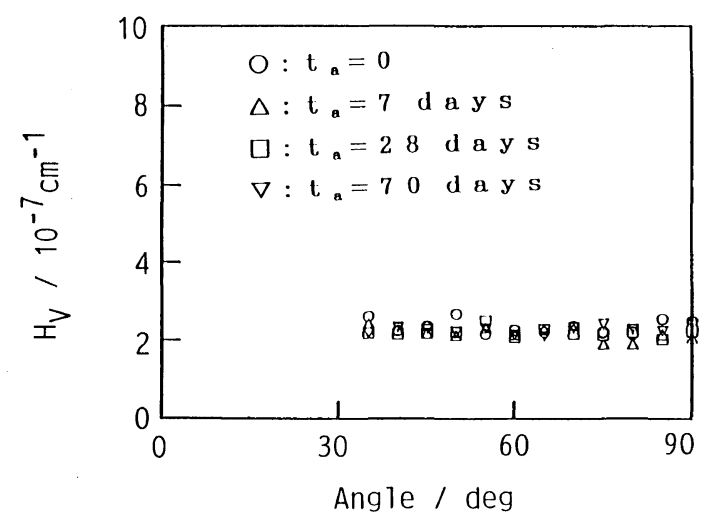

Figure 5. $H_{\mathrm{V}}$ scattering by PMMA glasses aged at $90^{\circ} \mathrm{C}$.

scattering for unaged and aged PMMA glasses. The $H_{\mathrm{V}}$ scattering was also independent of both aging time and scattering angle, and anisotropic light scattering loss $\alpha^{\text {aniso }}$ was about $2.7 \mathrm{~dB} \mathrm{~km}^{-1}$ at any aging time. This result means that the densification during aging below $T_{\mathrm{g}}$ does not generate the large-size isotropic or anisotropic heterogeneities and occurs homogeneously. It is seen that light scattering loss of PMMA glass is not affected inherently by physical aging.

PMMA glasses polymerized below $T_{\mathrm{g}}$ had excess scattering loss (hundreds $\mathrm{dB} \mathrm{km}^{-1}$ ) due to large-size (several hundreds $\AA$ ) heterogeneous structure with the order of $10^{-5}-10^{-4}$ of refractive-index difference. It was experimentally and theoretically clarified that this fluctuation would not be caused by a small amount of remaining monomers or additives, the molecular weight of polymers, the stereoregularity due to the specific tacticity of PMMA, nor crosslinking as the result of the gel effect. ${ }^{7}$

We investigated the effect of the heat treatment below and above $T_{\mathrm{g}}$ on the $V_{\mathrm{v}}$ intensity. ${ }^{7}$ It is quite interesting to point out that when PMMA glass polymerized below $T_{\mathrm{g}}$ was continuously heated below $T_{\mathrm{g}}$, the $V_{\mathrm{v}}$ intensity gradually increased, decreasing the remaining monomer. However, when PMMA glass polymerized below $T_{\mathrm{g}}$ was heat treated above $T_{\mathrm{g}}$, the $V_{\mathrm{v}}$ intensity remarkably decreased to the value predicted by the thermally induced fluctuation theory. Thus we can say that the $T_{\mathrm{g}}$ is the critical temperature for determining the excess scattering. Furthermore, the temperature dependence of the $V_{\mathrm{V}}$ intensity around $T_{\mathrm{g}}$ was investigated. ${ }^{8}$ As for low-loss PMMA glass with no excess scattering, the $V_{\mathrm{v}}$ versus temperature was quite consistent with the fluctuation theory. On the other hand, in the case of PMMA glass with excess scattering, an abrupt decrease in $V_{\mathrm{V}}$ scattering around $T_{\mathrm{g}}$ was observed with increasing temperature.

A slight amount of decrease in the remaining monomer at high conversion polymerization below $T_{\mathrm{g}}$ brought about extreme increase in the light scattering loss to several hundreds $\mathrm{dB} \mathrm{km}^{-1}$. In the case of PMMA, the volume contraction in the polymerization process is about $20 \%$. The excess scattering is, we believe, mainly due to voids caused by the volume shrinkage accompanying polymerization of the remaining monomer trapped inside the polymer glass. We proposed an excess scattering model ${ }^{11}$ at high conversion from the assumption that the heterogeneous structure consists of two phases due to the localization of the monomer, initiator, and voids caused by shrinkage during polymerization. This excess scattering model proposed shows that if a slight amount of monomer trapped inside the PMMA glass is polymerized in situ, generating slightly localized voids due to the aggregation of the unreacted initiator, a $1 \mathrm{wt} \%$ rise in the conversion around $P=95 \mathrm{wt} \%$ increases the scattering loss by several hundreds $\mathrm{dB} \mathrm{km}^{-1}$.

\section{CONCLUSION}

Isothermal aging has been carried out on low-loss PMMA glass at $18^{\circ} \mathrm{C}$ below $T_{\mathrm{g}}$ as the polymer approaches equilibrium. The effect of enthalpy relaxation on light scattering loss of PMMA glass which had no excess scattering was investigated by differential scanning calorimetry, density and light scattering measurements. The light scattering intensity of PMMA glass did not increase with increasing aging time. It is seen that light scattering loss of PMMA glass is not affected by physical aging under the above conditions.

\section{REFERENCES}

1. L. C. E. Struik, "Physical Aging in Amorphous Polymers and Other Materials," Elsevier, Amsterdam, 1978.

2. A. J. Kovacs, J. Polym. Sci., 30, 131 (1958).

3. R. Greiner and F. R. Schwarzl, Rheol. Acta, 23, 378 (1984).

4. H. Yoshida and Y. Kobayashi, Polym. J., 14, 925 (1982).

5. J. M. G. Cowie and R. Ferguson, Polymer, 34, 2135 (1993).

6. S. E. B. Petrie, J. Macromol. Sci., Phys., B12, 225 (1976).

7. Y. Koike, N. Tanio, and Y. Ohtsuka, Macromolecules, 22, 1367 (1989).

8. N. Tanio, Y. Koike, and Y. Ohtsuka, Polym. J., 21, 119 (1989).

9. N. Tanio, Y. Koike, and Y. Ohtsuka, Polym. J., 21, 259 (1989).

10. N. Tanio and Y. Koike, Kobunshi Ronbunshu, 53, 682 (1996) [in Japanese].

11. Y. Koike, S. Matsuoka, and H. E. Bair, Macromolecules, 25, 4807 (1992).

12. A. Einstein, Ann. Phys., 33, 1275 (1910).

13. P. Debye and A. M. Bueche, J. Appl. Phys., 20, 518 (1949).

14. P. Debye, H. R. Anderson, and H. Brumerger, J. Appl. Phys., 28, 679 (1957).

15. M. J. Bowden, E. A. Chandross, and I. P. Kaminow, Appl. Opt., 13, 112 (1974).

16. E. Muzeau, G. Vigier, and R. Vassoille, J. Non-Cryst. Solids, 172-174, 575 (1994). 